\title{
A new beam alignment method in SEM based on parallax principle
}

\author{
Luyang $\operatorname{Han}^{1}$, Markus Boese ${ }^{1}$, Bjoern Gamm ${ }^{1}$ and Benjamin Tordoff ${ }^{2}$
}

${ }^{1}$ Carl Zeiss Microscopy GmbH, Oberkochen, Germany, ${ }^{2}$ Carl Zeiss Microscopy GmbH, Germany

Autofocus is one of the most desirable function for everyday SEM usage. Although this function has been developed long time ago, there are still constant innovations and improvements to fulfill specific applications. Traditional autofocus type relies on the measurement of image sharpness from a scanning SEM image or profile. The autofocus method then optimizes the focus and other parameters on the SEM to maximize the measured sharpness [1]. This means the specimen needs to be scanned constantly to monitor the changes in sharpness when adjusting the focus, which leads to low speed and high electron doses on the focused area. Also, the chosen sharpness measure for the auto focus might not be universally applied to all kinds of samples, especially when the contrast on the sample shows preferred orientation or the sample might easily be modified by the electron beam. Most of commercial SEM use such auto focus method and suffers from the afore mentioned limitations. In this contribution we report a new autofocus method based on measuring the defocus of specimen through parallax effect, which does not rely on measuring the image sharpness. The principle of parallax autofocus is shown in Figure 1. By tilting of the primary electron beam against a defined pivot point, 2 SEM images can be obtained. Both images are blurred due to defocus, but the image shift can be evaluated very accurately using cross-correlation. As the tilt angle is known, using trigonometry one can easily derive the defocus value from the apparent image shift. Since only 2 consecutive SEM images are necessary, this autofocus method is extremely fast and minimum doses are applied on the sample. As both images are taken in slightly defocused state, this also minimizes the sample damage such as contaminations and charging artifacts. The fast speed and low sample damage make this method especially useful for automated workflow in SEM. The same parallax principle can also be extended to automatically correct for deviations in stigmator and aperture alignment. By combining tilting in different direction and angles, the complete beam can be aligned by taken as little as 8 images in total. In this contribution we will discuss this new beam alignment strategy in detail to show its advantages and limitations. Several typical use cases will also be discussed.

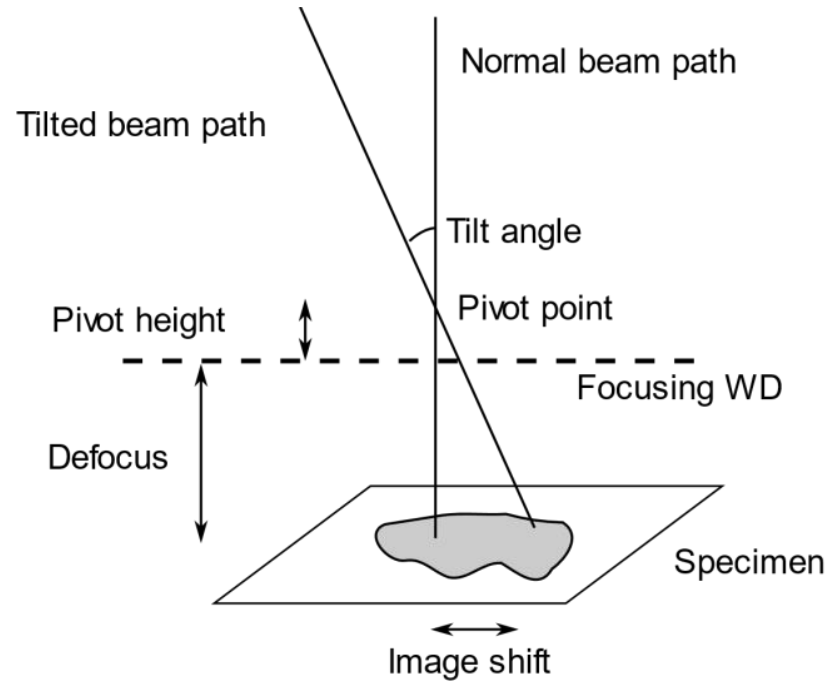

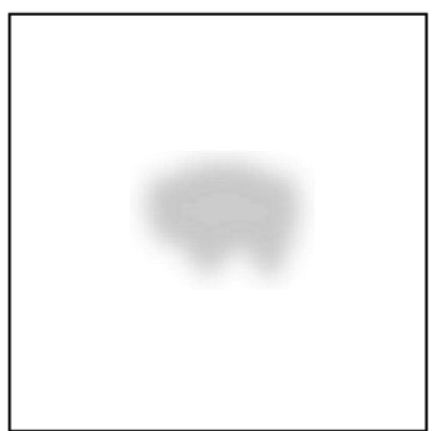

SEM image from normal beam path

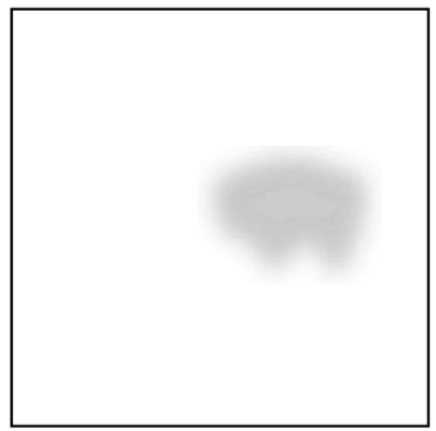

SEM image from tilted beam path 
Figure 1. 2 SEM images are obtained in normal beam path and tilted beam path. From the apparent shift between 2 images and known tilt angle, the defocus value can be calculated.

\section{References}

[1] L. Reimer, Scanning Electron Microscopy, 2nd. Edition (Springer), p 50 